\title{
PULSED EDDY-CURRENT DEVELOPMENT WITH APPLICATIONS TO THE NONDESTRUCTIVE EVALUATION OF MATERIALS
}

J. E. Coulter

\section{UNION CARBIDE CORPORATION}

\section{NUCLEAR DIVISION}

\section{OAK RIDGE Y-12 PLANT}

operated for the ATOMIC ENERGY COMMISSION under U. S. GOVERNMENT Contract W-7405 eng 26

UNION CARBIDE
OAK RIDGE Y-12 PLANT

P. O. Box $Y$

OAK RIDGE, TENNESSEE 37830 


\section{DISCLAIMER}

This report was prepared as an account of work sponsored by an agency of the United States Government. Neither the United States Government nor any agency Thereof, nor any of their employees, makes any warranty, express or implied, or assumes any legal liability or responsibility for the accuracy, completeness, or usefulness of any information, apparatus, product, or process disclosed, or represents that its use would not infringe privately owned rights. Reference herein to any specific commercial product, process, or service by trade name, trademark, manufacturer, or otherwise does not necessarily constitute or imply its endorsement, recommendation, or favoring by the United States Government or any agency thereof. The views and opinions of authors expressed herein do not necessarily state or reflect those of the United States Government or any agency thereof. 


\section{DISCLAIMER}

Portions of this document may be illegible in electronic image products. Images are produced from the best available original document. 
Printed in the United States of America. Available from

National Technical Information Service

U.S. Department of Commerce

5285 Port Royal Road, Springfield, Virginia 22151

Price: Printed Copy \$3.00; Microfiche \$0.95

This report was prepared as an account of work sponsored by the United States Government. Neither the United States nor the United States Atomic Energy Commission, nor any of their employees, nor any of their contractors, subcontractors, or their employees, makes any warranty, express or implied, or assumes any legal liability or responsibility for the accuracy, completeness or usefulness of any information, apparatus, product or process disclosed, or represents that its use would not infringe privately owned rights.

Reference to a company or product name does not imply approval or recommendation of the product by Union Carbide Corporation or the U S Atomic Energy Commission to the exclusion of others that may meet specifications. 


\title{
PULSED EDDY-CURRENT DEVELOPMENT WITH APPLICATIONS TO THE NONDESTRUCTIVE EVALUATION OF MATERIALS
}

\author{
J. E. Coulter
}

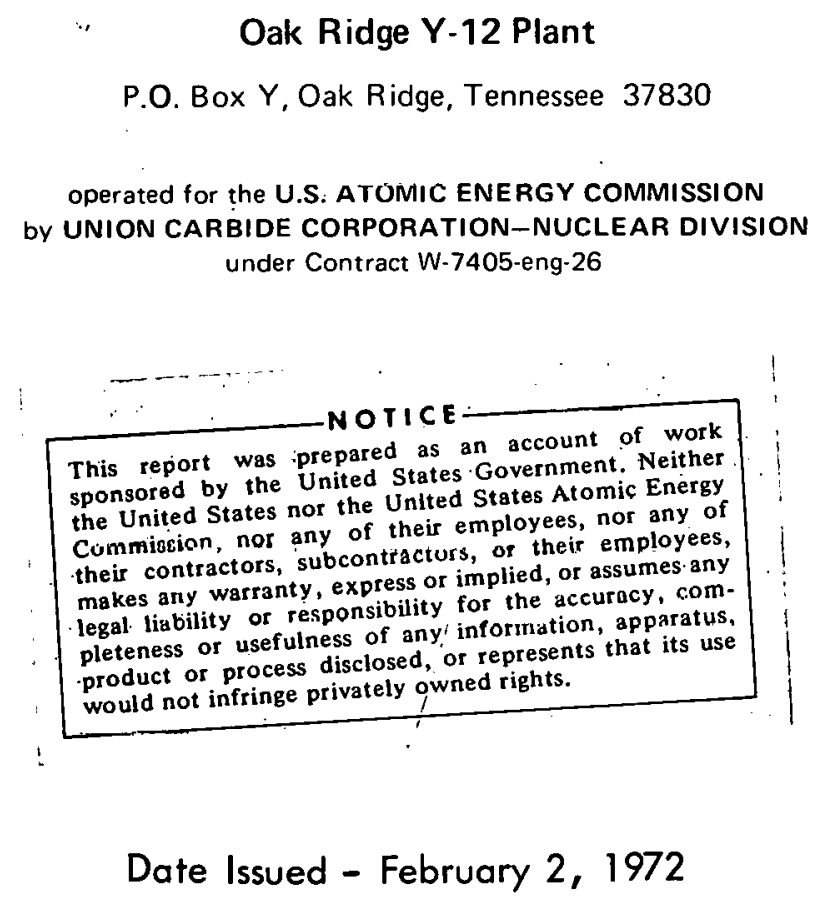




\section{DISTRIBUTION}

Atomic Energy Commission

Keller, C. A.

Zachry, D. S., Jr

Dow-Rocky Flats

Seed, J. R.

Lawrence Livermore Laboratory

MacMillan, K.

Stuart, F. R.

Taylor, G. M.

Vitale, J.K.

Los Alamos Scientific Laboratory

Elliot, D. E.

Oak Ridge Gaseous Diffusion Plant

Arendt, J.

Jordan, R. G.

Wilcox, W. J., Jr

Oak Ridge National Laboratory

Dodd, C. V.

Oak Ridge $Y-12$ Plant

Arnold, J. G.

Bernander, N.K.

Briscoe, O.W.

Burditt, R. B.

Burkhart, L. E.
Cadden, J. L.

Canary, W. W.

Coulter, J. E. (5)

Denny, A. (2)

Dodson, W. H.

Franco-Ferreira, E. A.

Garber, J. W.

Hemphill, L. F.

Hulsey, W. J.

Jackson, V.C.

Kahl, K. G.

Keith, Alvin

King, J. F.

Long, $P . J$.

Mason, D. L.

McLendon, J. D.

Mitchel, G.W.

Moyer, M. W.

Mundt, F. D.

Ross, W. D.

Schreyer, J. M.

Smith, J. H.

Smith, R. F.

Snow, S. G.

Speller, B. W.

Tullos, E.J.

Turner, P. W.

Weathersby, W. E.

Wesley, R. L.

Wright, C. C.

Yaggi, W. J.

Y-12 Central Files (5)

$Y-12$ Central Files (master copy)

$Y-12$ Central Files (route)

$Y-12$ Central Files $(Y-12 R C)$

Paducah Gaseous Diffusion Plant

Winkel, R. A. 
Sandia-Albuquerque

Ballard, D. W.

Burchett, O.J.

Sandia-Livermore

Baker, A. F.

In addition, this report is distributed in accordance with the category UC-4, Chemistry, as given in the "USAEC Standard Distribution Lists for Unclassified Scientific and Technical Reports", TID-4500. 
THIS PAGE

\section{WAS INTENTIONALLY \\ LEFT BLANK}




\section{ABSTRACT}

A pulsed eddy-current system has been developed for use in the nondestructive evaluation of materials. A qualitative theory is presented in conjunction with experimental data to support some of the basic results of this theory. 


\section{CONTENTS}

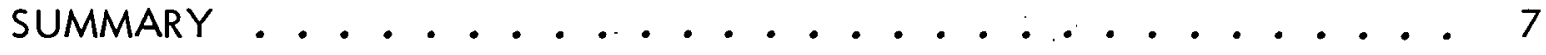

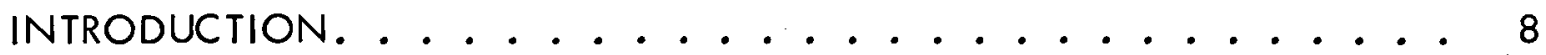

PULSED EDDY-CURRENT DEVELOPMENT WITH APPLICATIONS TO THE NONDESTRUCTIVE EVALUATION OF MATERIALS ....... 9

Theory ............................. 9

Experimental Methods................. . 16

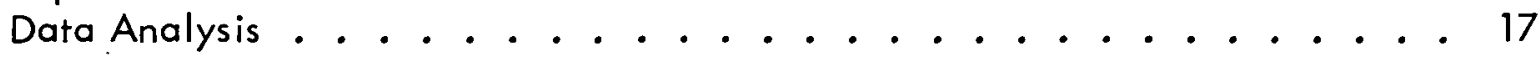

Discussion...................... 24

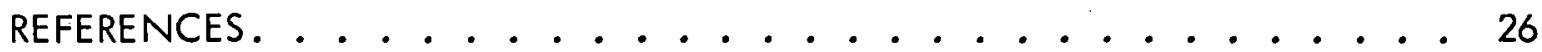

BIBLIOGRAPHY ..................... 27 
A pulsed eddy-current system has been developed primarily for use in the testing of welds in several different materials. However, to attain a better understanding of the nature of the pulsed-current field and the parameters which affect pulse shape, a theoretical analysis was made for the case of a finite pulsed magnetic field applied at the surface of a conductor in which it was shown that the pulse width should be proportional to the conductivity, thickness, and permeability of the conductor. Experimental data were taken to support these theoretical conclusions, and additional data are presented to demonstrate the application of the pulsed system to the nondestructive testing of materials. Particular emphas is is placed upon the use of sampling techniques which enable one to choose the point on the pulse which contains the most information concerning the variable to be tested. A lift-off compensating servo-system is described which makes use of one of these sampling methods to provide the pulsed system with a continuous scanning capability. 


\section{INTRODUCTION}

A relatively recent development in the field of eddy-current nondestructive testing is the use of pulsed currents rather than the more conventional steady-state, sinusoidal currents. The transient nature of the pulsed-current field allows the use of signal-processing methods which were not applicable to steady-state, eddy-current testing. Since more information is contained in a single pulsed signal, . sampling techniques can be applied to select the particular point (in time) on the pulse which contains the most information concerning the variable to be tested. Better thermal stability and higher resolution are distinct advantages of the pulsed eddy-current system over steady-state eddy-current systems.

To provide a noncontact method of testing the penetration depth of electron-beam welds in several different materials, a pulsed eddy-current system was constructed and successfully tested for this application at the Oak Ridge $Y-12$. Plant. (a) However, at the same time, a more basic analys is of the pulsed system was made to attain a better understanding of the nature of these pulsed currents and the parameters which affect the pulse shape. A qualitative theory was constructed and experimental data were taken to support some of the theoretical results. No attempt was made to derive a quantitative theory which was representative of the actual experimental conditions; but; instead, the emphasis was placed on the experimental confirmation of some of the basic theoretical conclusions and the capability of the pulsed eddycurrent system as a reliable nondestructive testing method.

(a) Operated for the US Atomic Energy Commission by the Union Carbide Corporation's Nuclear Division. 


\section{PULSED EDDY-CURRENT DEVELOPMENT WITH APPLICATIONS} TO THE NONDESTRUCTIVE EVALUATION OF MATERIALS

\section{THEORY}

The following plane-wave analys is of an electromagnetic field in a two-conductor medium is similar to the analysis presented by D. L. Waidelich, et al. (1) However, the theory presented herein has been extended to include an input step magnetic field having a finite time width.

If $\bar{E}$ and $\bar{H}$ are the Laplace transforms of the electric and magnetic field intensities, respectively, then Maxwell's equations take the following form for a material conductor:

$$
\begin{gathered}
\vec{\nabla} \cdot \overline{\mathrm{E}}=0, \\
\vec{\nabla} \cdot \overline{\mathrm{H}}=0, \\
\vec{\nabla} \times \overline{\mathrm{H}}=\sigma \overline{\mathrm{E}}, \\
\vec{\nabla} \times \overline{\mathrm{E}}=-\mu \mathrm{s} \overline{\mathrm{H}},
\end{gathered}
$$

where $\sigma$ and $\mu$ are the conductivity and permeability, respectively, of the medium, and $s$ is the complex variable of the Laplacian transform. Figure 1 shows the case of the plane wave in normal incidence upon the medium and traveling in the $z$-direction, where the $E$ field is in the $x$-direction and the $H$ field is in the $y$-direction.

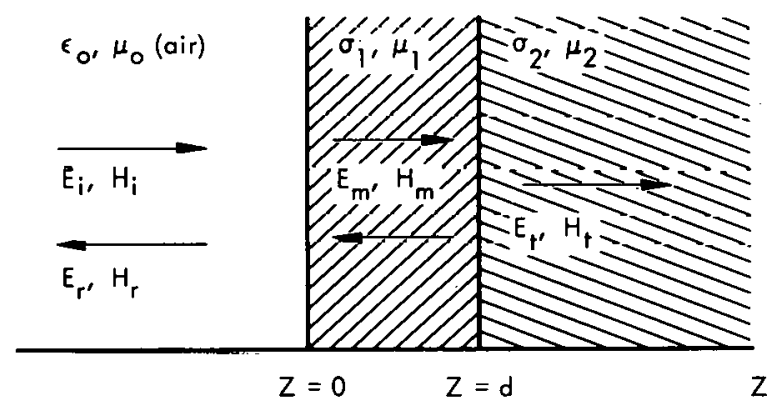

Figure 1. PLANE WAVE IN NORMAL INCIDENCE UPON A TWO-CONDUCTOR MEDIUM.

It can be shown from Equation 1 that for a material conductor:

$$
\frac{d^{2} H_{y}}{d z^{2}}=s \mu \sigma H_{y} \text {. }
$$


Therefore:

$$
H_{y}=A e^{-\sqrt{s \mu \sigma} z}+B e^{+\sqrt{s \mu \sigma} z} .
$$

Also,

$$
E_{X}=-\frac{1}{\sigma} \cdot \frac{d H_{y}}{d z}=\sqrt{\frac{s \mu}{\sigma}}\left[A e^{-\sqrt{s \mu \sigma} z}-B e^{+\sqrt{s \mu \sigma} z}\right]
$$

Similarly, in air:

$$
\frac{d^{2} H_{y}}{d z^{2}}=s^{2} \epsilon_{0} \mu_{0} H_{y}
$$

Therefore:

$$
H_{y}=C e^{-s \sqrt{\epsilon_{0} \mu_{0}} z}+D e^{+s \sqrt{\epsilon_{0} \mu_{0}} z} .
$$

Also,

$$
E_{x}=-\frac{1}{s \epsilon_{0}} \frac{d H_{y}}{d z}=\sqrt{\frac{\mu_{0}}{\epsilon_{0}^{\circ}}}\left[C e^{-s \sqrt{\epsilon_{0} \mu_{0}} z}-D e^{+s \sqrt{\epsilon_{0} \mu_{0}} z}\right] .
$$

Let

$$
\begin{gathered}
H_{i}=H_{0} e^{-s \sqrt{\epsilon_{0} \mu_{0} z},} \\
H_{r}=H_{1} e^{+s \sqrt{\epsilon_{0} \mu_{0}} z}, \\
H_{m}=H_{2}^{-} e^{-\sqrt{s \mu_{1} \sigma_{1}} z}+H_{2}^{+} e^{+\sqrt{s \mu_{1} \sigma_{1}} z}, \text { and } \\
H_{t}=H_{3} e^{-\sqrt{s \mu_{2} \sigma_{2}} z} .
\end{gathered}
$$

Applying the boundary conditions at $z=0$, then: 


$$
\begin{array}{r}
H_{0}+H_{1}=H_{2}^{-}+H_{2}^{+} \text {, and } \\
Z_{0}\left(H_{0}-H_{1}\right)=Z_{1}\left(H_{2}^{-}-H_{2}^{+}\right),
\end{array}
$$

where:

$$
Z_{0}=\sqrt{\frac{\mu_{0}}{\epsilon_{0}}} \text { and } Z_{1}=\sqrt{\frac{s \mu_{1}}{\sigma_{1}}}
$$

Similarly at $z=d:$

$$
\begin{gathered}
H_{2}^{-} e^{-\sqrt{s \mu_{1} \sigma_{1}} d}+H_{2}^{+} e^{+\sqrt{s \mu_{1} \sigma_{1}} d}=H_{3} e^{-\sqrt{s \mu_{2} \sigma_{2}} d} \text {, and } \\
Z_{1}\left(H_{2}^{-} e^{-\sqrt{s \mu_{1} \sigma_{1}} d}-H_{2}^{+} e^{+\sqrt{s \mu_{1} \sigma_{1}} d}\right)=Z_{2} H_{3} e^{-\sqrt{s \mu_{2} \sigma_{2}} d},
\end{gathered}
$$

where:

$$
z_{2}=\sqrt{\frac{s \mu_{2}}{\sigma_{2}}}
$$

Defining $Z_{i k}=\frac{Z_{k}}{Z_{i}}$, then Equations 12, 13, 14, and 15 take the following form:

$$
\begin{gathered}
H_{0}+H_{1}=H_{2}^{-}+H_{2}^{\prime l}, \\
H_{0}-H_{1}=Z_{01}\left(H_{2}^{-}-H_{2}^{+}\right), \\
H_{2}^{-e} \sqrt{s \mu_{1} \sigma_{1}} d H_{2}^{\prime} e^{+\sqrt{s \mu_{1} \sigma_{1}} d}=H_{3} e^{-\sqrt{s \mu_{2} \sigma_{2}} d}, \text { and } \\
H_{2}^{-} e^{-\sqrt{s \mu_{1} \sigma_{1}} d}-H_{2}^{+}{\sqrt{s \mu_{1} \sigma_{1}} d}^{+\sqrt{s \mu_{2} \sigma_{2}} d} .
\end{gathered}
$$

Solving for the ratio of the amplitudes of the reflected and incident waves, then: 


$$
\frac{H_{1}}{H_{0}}=\frac{\frac{\left(1-Z_{01}\right)}{\left(1+Z_{01}\right)}+\frac{\left(1-z_{12}\right)}{\left(1+Z_{12}\right)} e^{-2 \sqrt{s \mu_{1} \sigma_{1}} d}}{1+\frac{\left(1-z_{01}\right)}{\left(1+Z_{01}\right)} \frac{\left(1-z_{12}\right)}{\left(1+Z_{12}\right)} e^{-2 \sqrt{s \mu_{1} \sigma_{1}} d}} .
$$

Define $R_{i k}=\frac{1-z_{j k}}{1+z_{j k}}=\frac{z_{j}-z_{k}}{z_{j}+z_{k}}$.

Therefore, the reflected wave in air from the surface of the conductor is given by:

$$
H_{1}=H_{0}\left[\frac{R_{01}+R_{12} e^{-2 \sqrt{s \mu_{1} \sigma_{1}} d}}{1+R_{01} R_{12} e^{-2 \sqrt{\mu_{1} \sigma_{1}} d}}\right] \text {, }
$$

which becomes after expansion:

$$
\begin{aligned}
& H_{1}=H_{0}\left[R_{01}+R_{12}\left(1-R_{01}^{2}\right) e^{-2 \sqrt{s \mu_{1} \sigma_{1}} d}-\right. \\
& \left.R_{01} R_{12}^{2}\left(1-R_{01}^{2}\right) e^{-4 \sqrt{s \mu_{1} \sigma_{1}} d}+\ldots\right] .
\end{aligned}
$$

Keeping only first-order terms in $R_{12}$ which is the ratio of the amplitudes of the reflected and incident waves at the metal-to-metal boundary at $z=d$, then one can approximate the reflected wave $\mathrm{H}_{12}$ at the metal-to-metal interface so that:

$$
H_{12} \simeq H_{0} R_{12} e^{-2 \sqrt{s \mu_{1} \sigma_{1}} d},
$$

where:

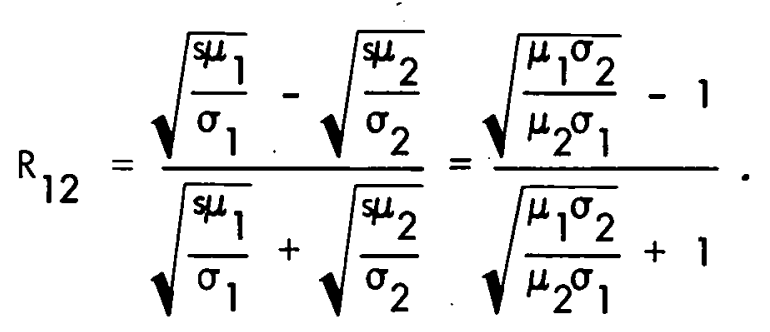




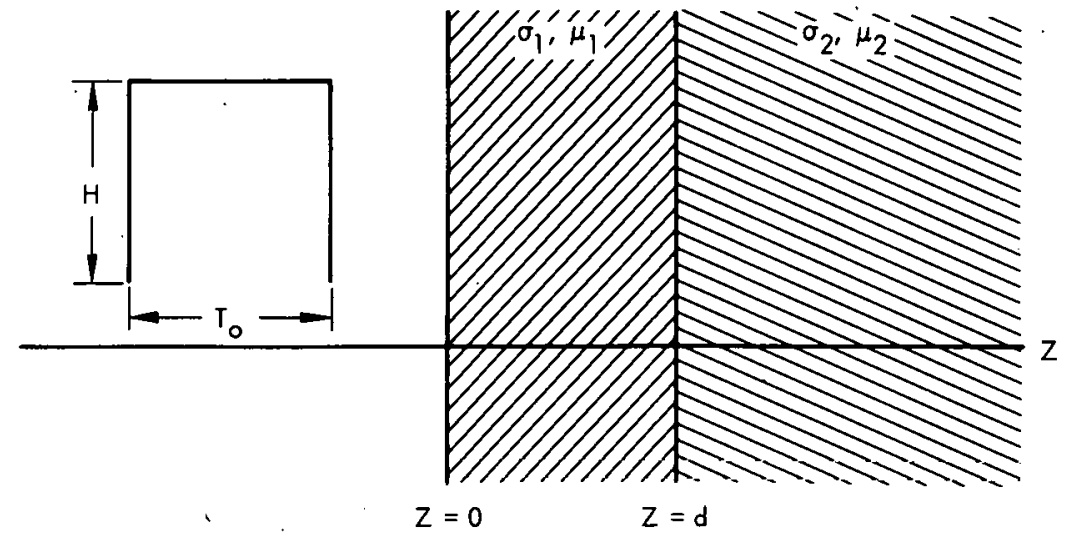
Figure 2. FINITE PULSE OF STRENGIH H AND DURATION T。 APPLIED AT THE
SURFACE OF A TWO-CONDUCTOR MEDIUM.

Now, as shown in Figure 2, let $\mathrm{H}_{0}=\mathrm{H}_{0}(\mathrm{~s})$ be a finite pulse of strength $\mathrm{H}$ and duration $T_{0}$ which is applied at the surface of the first conductor $(z=0)$. at $t=0$; therefore,

$$
H_{0}(s)=H \frac{\left[1-e^{-s T_{0}}\right]}{s T_{0}},
$$

but:

$$
\frac{\left[1-e^{-s T_{0}} 0\right]}{s T_{0}}=1-\frac{s T_{0}}{2}+\frac{{ }^{2} T_{0}^{2}}{3 !}-\ldots+\frac{(-1)^{n}\left(s T_{0}\right)^{n}}{(n+1) !}+\ldots
$$

Keeping only first-order terms in $\mathrm{sT}_{0}$, then:

$$
H_{0}(s) \simeq H\left[1-\frac{s T}{2}\right],
$$

and

$$
H_{12} \simeq H_{12}\left[1-\frac{s T_{0}}{2}\right] e^{-a \sqrt{s}},
$$

where:

$$
a=2 d \sqrt{\sigma_{1} \mu_{1}}
$$

Taking the inverse Laplace transform of Equation 26, then: 


$$
H_{12} \simeq H R_{12} \frac{a}{2 \sqrt{\pi}} e^{-\frac{a^{2}}{4 t}}\left[-T_{0} \frac{a^{2}}{8} t^{-7 / 2}+\frac{3}{4} T_{0} t^{-5 / 2}+t^{-3 / 2}\right] .
$$

Since the output of the pulsed system will be in terms of a voltage across acoil, then the time derivative of the magnetic field should be proportional io the output voltage; thus, taking the time derivative of Equation 27 yields:

$$
\begin{gathered}
\frac{d H_{12}}{d t} \simeq H R_{12} \frac{a}{2 \sqrt{\pi}} e^{-\frac{a^{2}}{4 t}}\left[-\frac{a^{4}}{32} T_{0} t^{-11 / 2}+\frac{5}{8} a^{2} T_{0}{ }^{-9 / 2}+\right. \\
\left.\left(\frac{a^{2}}{4}-\frac{15}{8} T_{0}\right) t^{-7 / 2}-\frac{3}{2} t^{-5 / 2}\right] .
\end{gathered}
$$

Now, if the conductivity $\sigma_{2}$ of the second conductor approaches zero (toward that of air), then $R_{12}$ approaches a value of $(-1)$.

Therefore, (dropping subscripts on $\sigma_{1}$ and $\mu_{1}$ ),

$$
\begin{gathered}
\frac{d H_{12}}{d t} \simeq H d \frac{\sqrt{\sigma \mu}}{\sqrt{\pi}} e^{-\frac{d^{2} \sigma \mu}{t}}\left[\frac{d^{4} \sigma^{2} \mu^{2}}{2} T_{0} t^{-11 / 2}-\frac{5 d^{2}}{2} \sigma \mu T_{0} t^{-9 / 2}+\right. \\
\left.\left(\frac{15}{8} T_{0}-d^{2} \sigma \mu\right) t^{-7 / 2}+\frac{3}{2} t^{-5 / 2}\right] .
\end{gathered}
$$

It is interesting to note that if $\mathrm{T}_{0}=0$, thus making the incident magnetic field $\mathrm{H}_{0}$ an impulse, then the crossing point of the pulse occurs at $T_{w}=\frac{2 d^{2}}{3} \sigma \mu$.

This result is similar to that obtained by $D$. L. Waidelich, ef al, $(1)$ where the input magnetic field was assumed to be an impulse with the result that the first crossing point of the pulse occurred at $T_{w}=0.367 d^{2} \sigma \mu$.

In Figure 3, the theoretical output voltage pulse, $\frac{d H_{12}}{d t}$, from Equation 29 is plotted as a function of time for the specific case of magnesium having a conductivity, $\sigma$, of $2.17 \times 10^{7} \mathrm{mhos} / \mathrm{meter}$ and a thickness, $d$, of 0.100 inch. For an input pulse width, $T_{0}=1$ millisecond, the first crossing point of the pulse occurs at 42 microseconds. For comparison, Figure 4 shows an oscilloscope photograph of the actual experimental pulse for the same thickness of magnesium, and the experimental pulse 


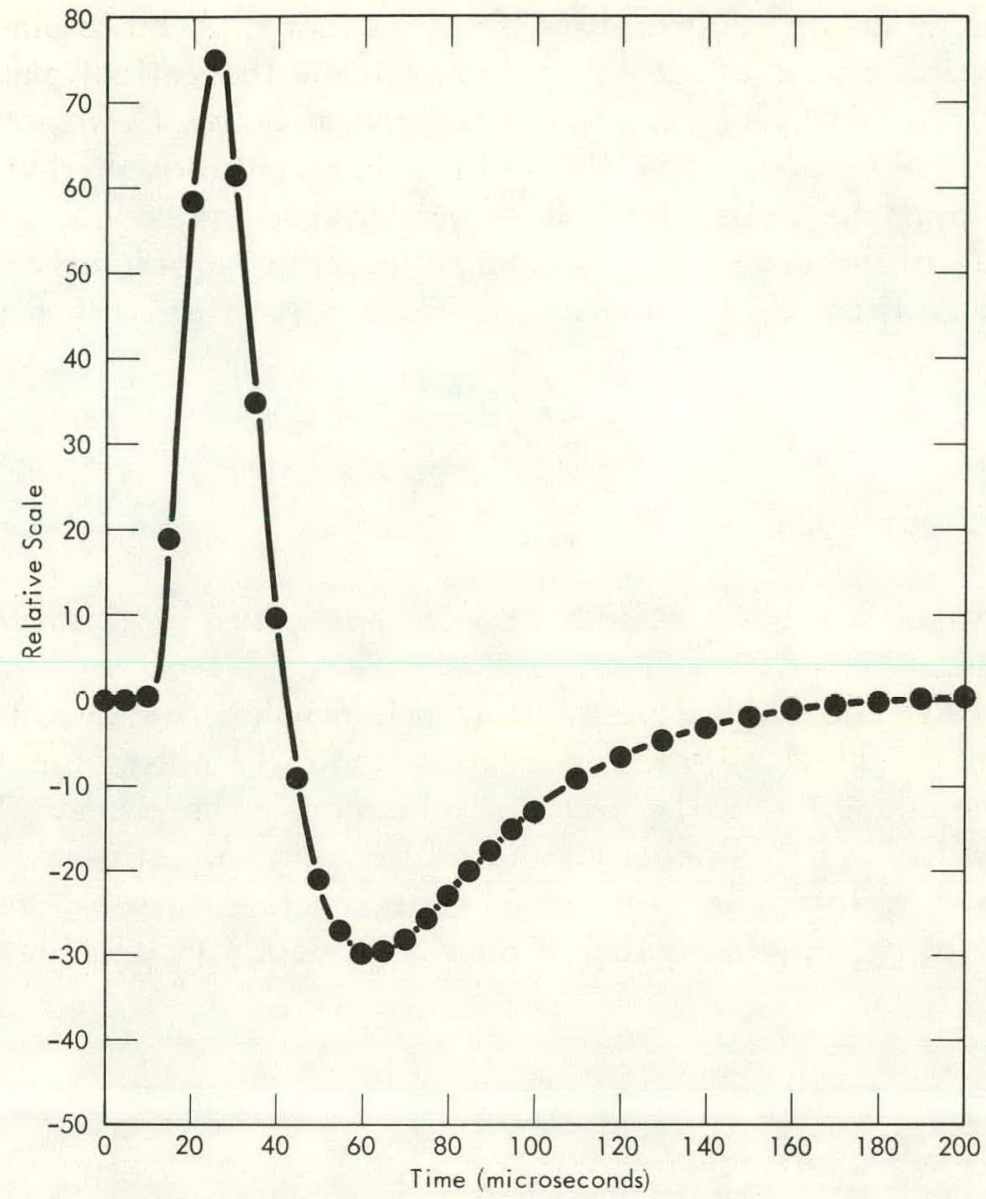

Figure 3. $\frac{\mathrm{dH}_{12}}{\mathrm{dT}}$ AS A FUNCTION OF TIME FOR MAGNESIUM. (The Mg Has a Conductivity of $2.17 \times 10^{7}$ Mhos/Meter and a Thickness of 0.100 Inch)

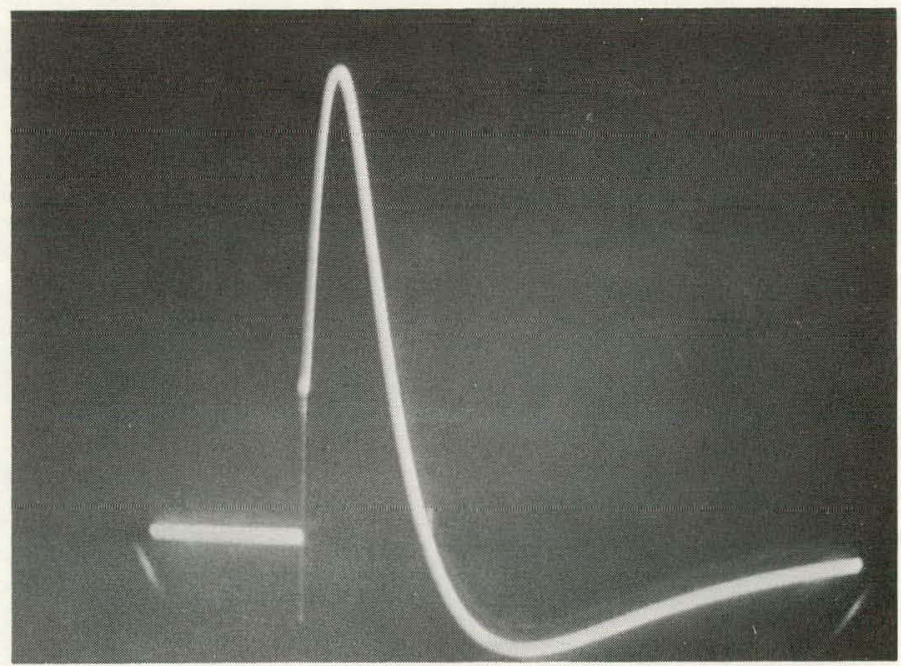

Figure 4. OSCILLOSCOPE PHOTOGRAPH OF THE EXPERIMENTAL VOLTAGE PULSE FOR MAGNESIUM. 
width was found to be approximately 47 microseconds. A basic similarity can be seen in the general shape of the two pulses, but the theoretical pulse has a slower rise time and a faster fall time than the experimental pulse. However, the important result of the preceding qualitative theory of a finite pulse applied at the surface of a conductor is that the pulse width is proportional to the conductivity, thickness, and permeability of the conductor; therefore, the crossing point of the pulse can be used to make quantitative measurements of these parameters with the aid of proper standards.

\section{EXPERIMENTAL METHODS}

A block diagram of the basic pulsed eddy-current system is illustrated in Figure 5. The pulse generator drives the primary of the pulse transformer with 20-volt,1-millisecond pulses. The secondary of the pulse transformer has its center tap grounded in order to provide equal amplitude but opposite polarity pulses for the two coaxial coils (the reference coil and the test coil) located in the probe. These two coils are electrically isolated from each other and form one arm of a bridge circuit with the attenuation circuit as the other arm of the bridge. Initially, the two coils are balanced in air (using the attenuator) to obtain (as nearly as possible) a null output.

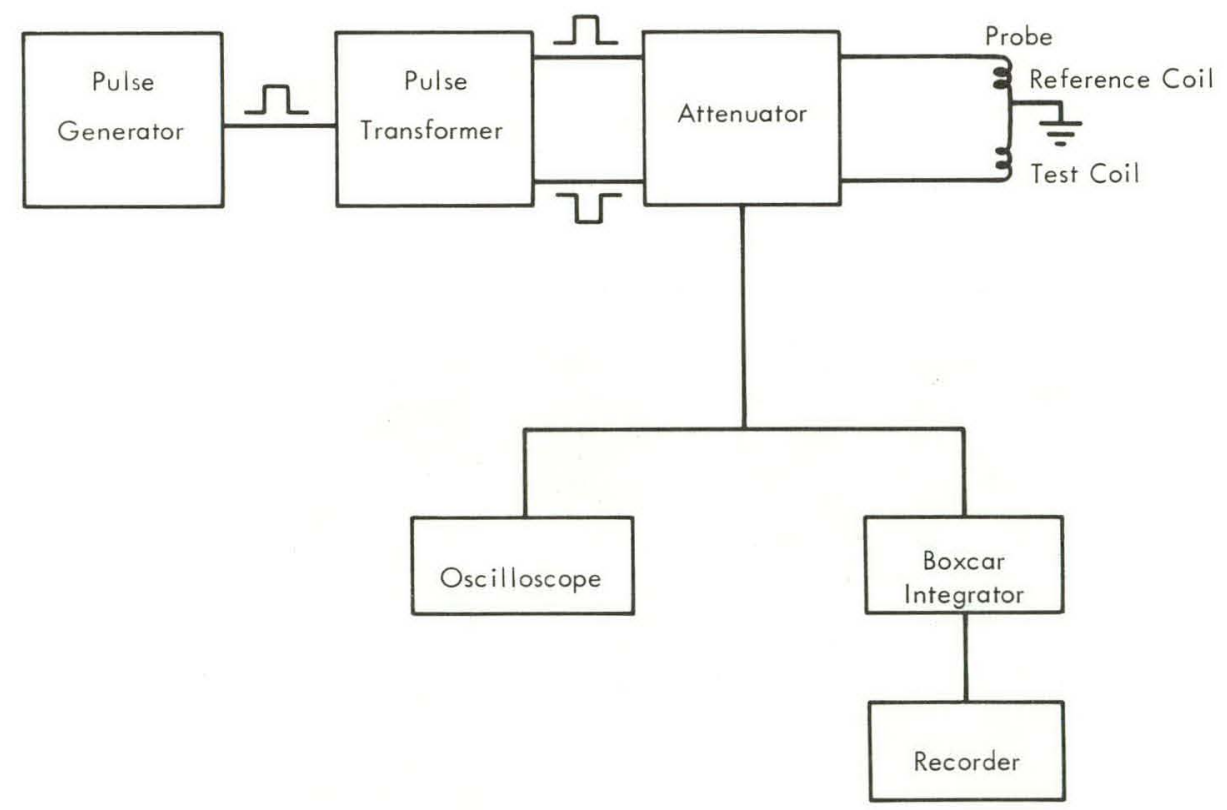

Figure 5. BLOCK DIAGRAM OF THE BASIC PULSED EDDY-CURRENT SYSTEM.

When the test coil is placed near a conductor, a difference voltage pulse is obtained at the output of the attenuator which is connected to a Boxcar Integrator and an oscilloscope. The sampling gate of the Boxcar Integrator provides the capability of 
scanning and selecting the point on the output pulse which contains the most information concerning the variable to be tested. This sampled output signal can then be used to drive a recorder or digital data acquisition system.

To maintain a constant probe-to-metal spacing (liftoff), a liftoff compensating servosystem was constructed to provide a continuous scanning capability for testing materials. The peak value of the output pulse, which is sensitive to liftoff, is sampled with the sampling unit of an oscilloscope, and this sampled signal is used to drive a closed-loop, servo-system which consists of a stepping motor, drive unit, and electronic switching. The motor and drive unit provide 1,000 steps per revolution of the shaft which is geared to 0.050 inch per revolution. This servo-system is capable of maintaining any desired probe-to-metal spacing (up to 0.1 inch) to within \pm 0.0005 inch. Figures 6 and 7 show the complete pulsed eddy-current system (including the liftoff compensating servo-system) with detailed connections.

\section{DATA ANALYSIS}

The theoretical analysis of a finite pulse applied at the surface of a conductor predicts that the pulse width is proportional to the conductivity, thickness, and permeability of the conductor. In order to establish these theoretical conclusions, several materials having different conductivities and thicknesses were investigated by varying these parameters (one at a time) to determine how these changes affect the shape of the pulse. The experimental graph in Figure 8 shows the effect of a change in conductivity upon pulse shape where liftoff and thickness were held constant. These pulses were plotted on an $X-Y$ recorder by scanning the contour of the pulse with the sampling gate of the Boxcar Integrator. Both pulse height and pulse width can be seen to decrease as the conductivity decreases. Equation 29 predicts the observed decrease in pulse width, but it also predicts that the pulse height will increase with a decrease in conductivity or thickness. However, Equation 29 only includes the "reflected" wave at the back surface of the conductor, whereas front surface "reflection" and interference terms were ignored in the derivation. Since the peak value of the pulse occurs earlier in time, this part of the pulse will be more sensitive to conditions near the surface of the conductor. The current density of a goodconductor is also expected to be greater near the surface of the conductor, a phenomenon analogous to the skin effect in steady-state, eddy-current testing.

In Figure 9, the output pulse is plotted for several different thicknesses of magnesium. As theoretically predicted, the width of the pulse decreases with a decrease in the thickness of the material. Since the conductivity and liftoff are held constant, the amplitude of the pulse remains the same for the different values of thickness. Perhaps the most interesting phenomenon of the pulsed current field is the effect of liftoff upon the shape of the pulse. As shown in Figure 10, the amplitude and slope of the pulse decrease as the probe-to-metal spacing is increased; however, the time width of the pulse remains constant for small changes in probe-to-metal spacing. Since the 


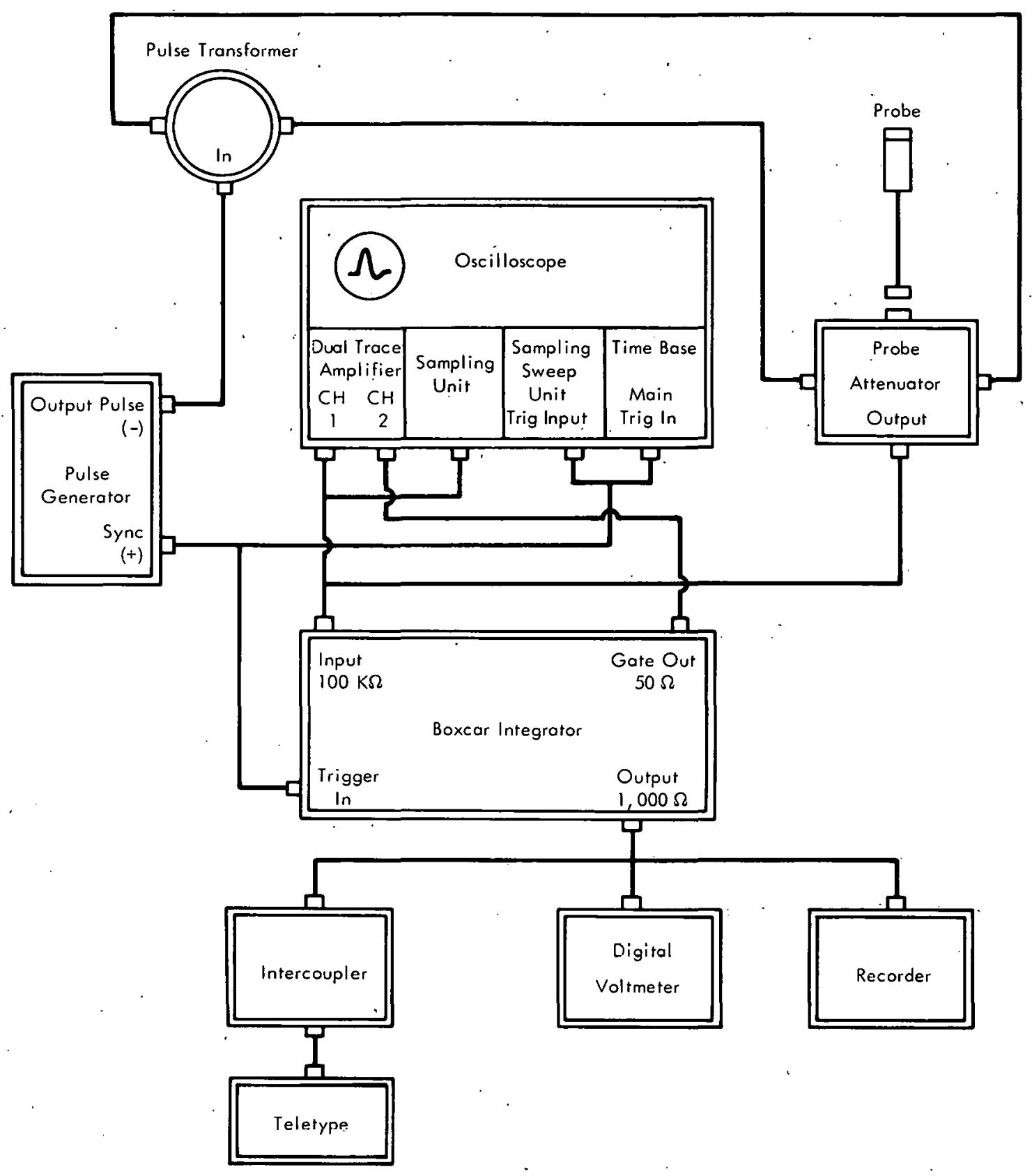

Figure 6. PULSED EDDY-CURRENT SYSTEM.

cross-over point of the pulse is relatively insensitive to liftoff, this particular point on the pulse can be used to make a thickness or conductivity measurement with the aid of suitable standards.

As mentioned previously, the pulsed eddy-current system was designed primarily to make noncontact measurements of the penetration of electron-beam welds. In particular, a partial penetration weld into a 0.150 -inch-thick aluminum butt joint was 


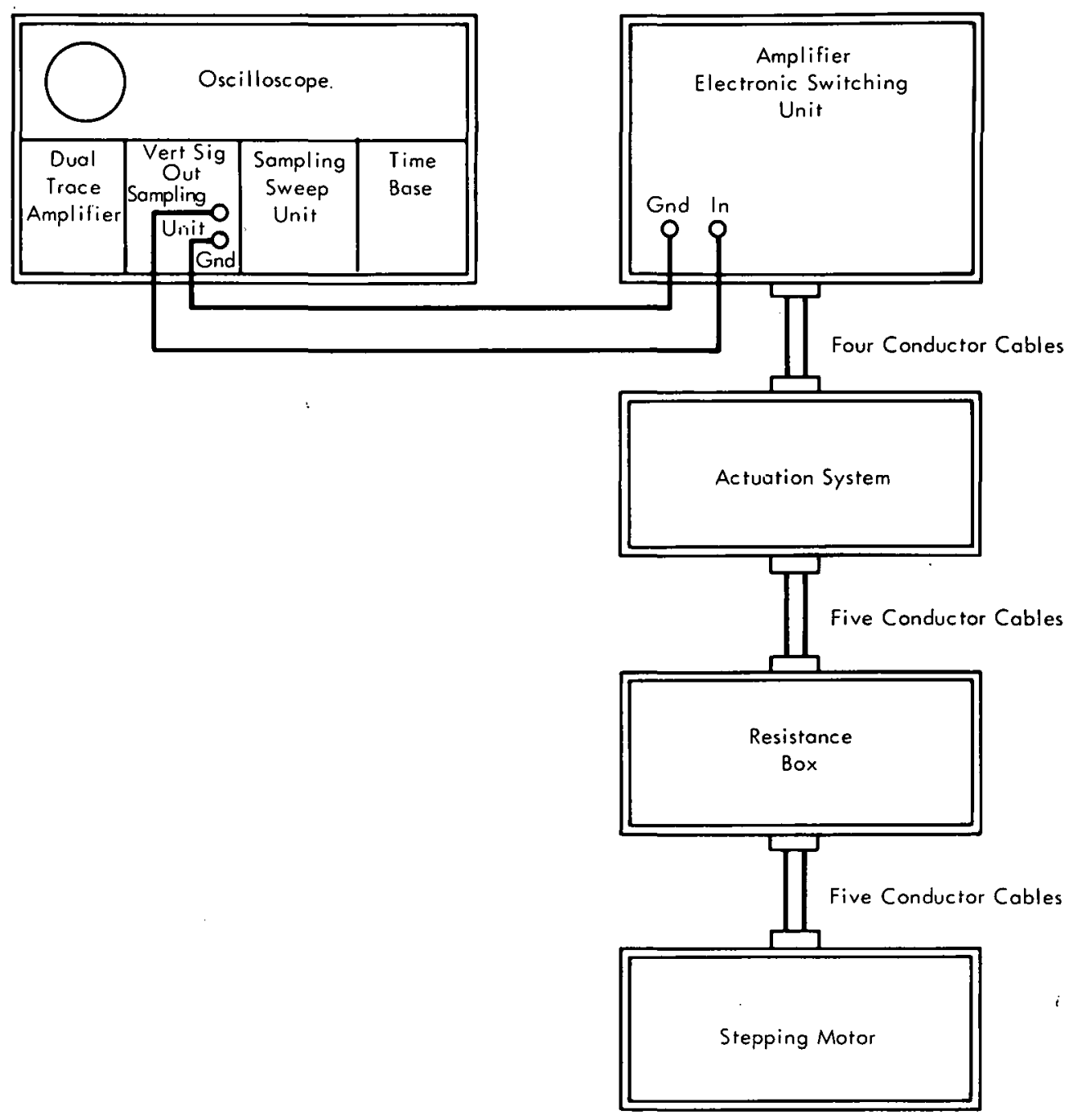

Figure 7. LIFTOFF COMPENSATING SERVO-SYSTEM.

tested using this system. Figure 11 shows the behavior of the pulse when the probe was positioned over the centerline of a weld having a partial penetration of 0.078 inch and for another weld whose penetration was 0.025 inch. A decrease in weld penetration causes a small decrease in the time width of the pulse; therefore, an increase in the amount of unfused joint beneath the weld appears to be characterized as a decrease in the equivalent thickness of the material in the weld region. These small changes in pulse width are more easily detected by first sampling the pulse at the cross-over point when the probe is positioned over the base material (off the weld). Then, when the probe is placed over the weld, a decrease in penetration is detected as a decrease in the DC level of the sampled output signal because the sampling gate is stationary in time and measures the pulse voltage at this point with respect to DC zero. In Figure 12, the penetration of four weld standards is plotted as a function of the sampled output signal. Liftoff is controlled by monitoring the 


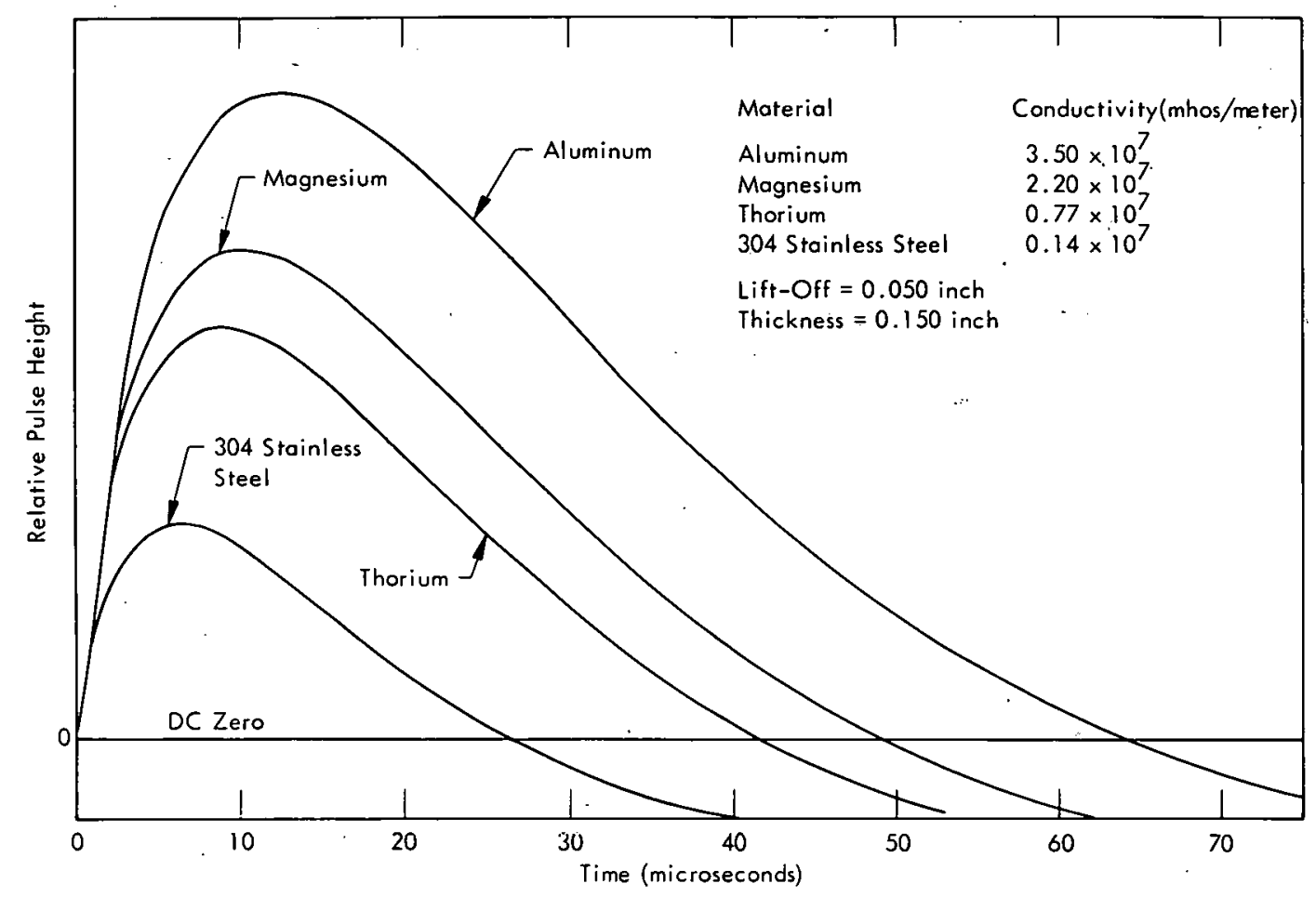

Figure 8. EXPERIMENTAL VOLTAGE PULSE FOR SEVERAL DIFFERENT MATERIAL CONDUCTORS.

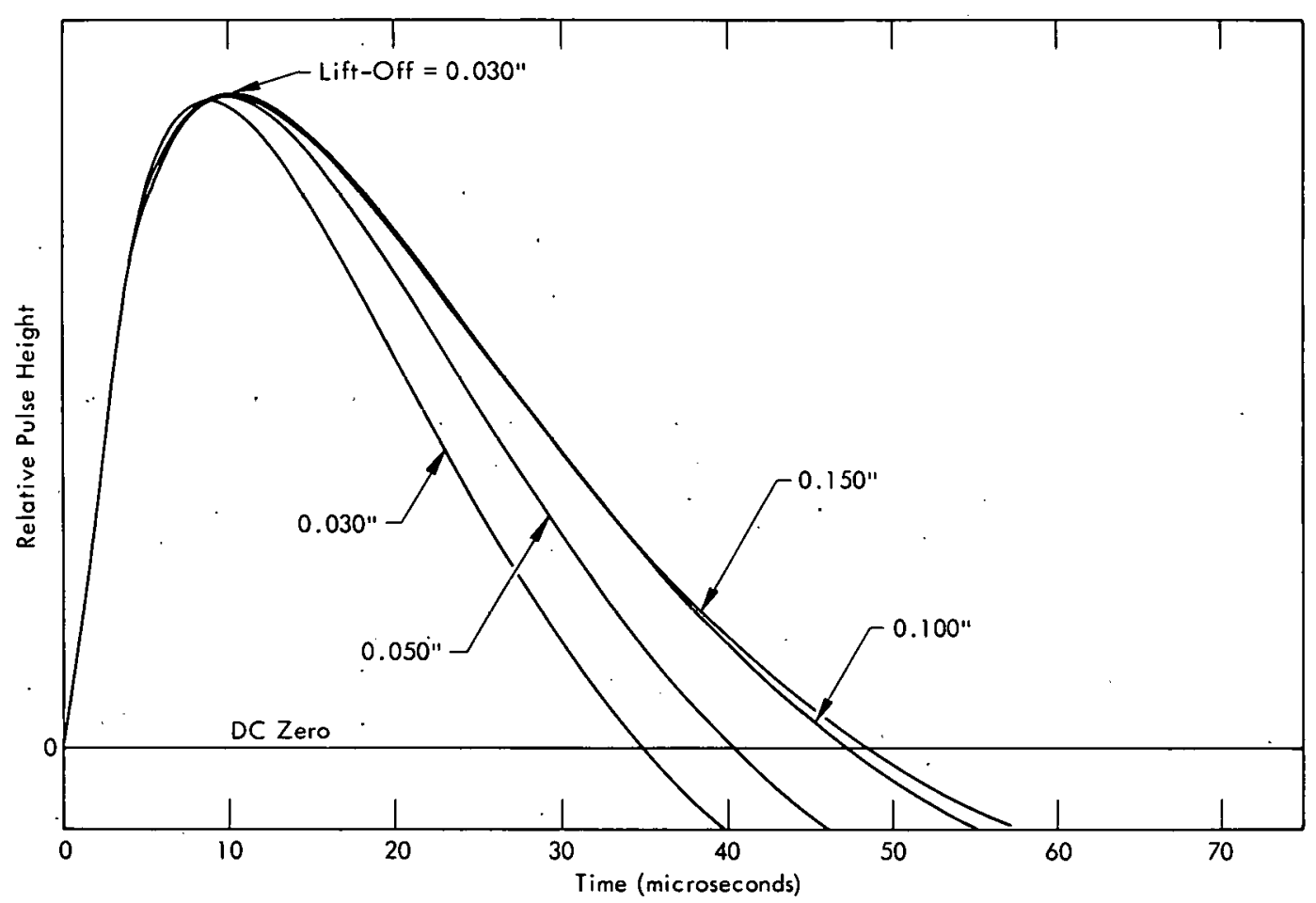

Figure 9. EXPERIMENTAL VOLTAGE PULSE FOR DIFFERENT THICKNESSES OF MAGNESIUM. 


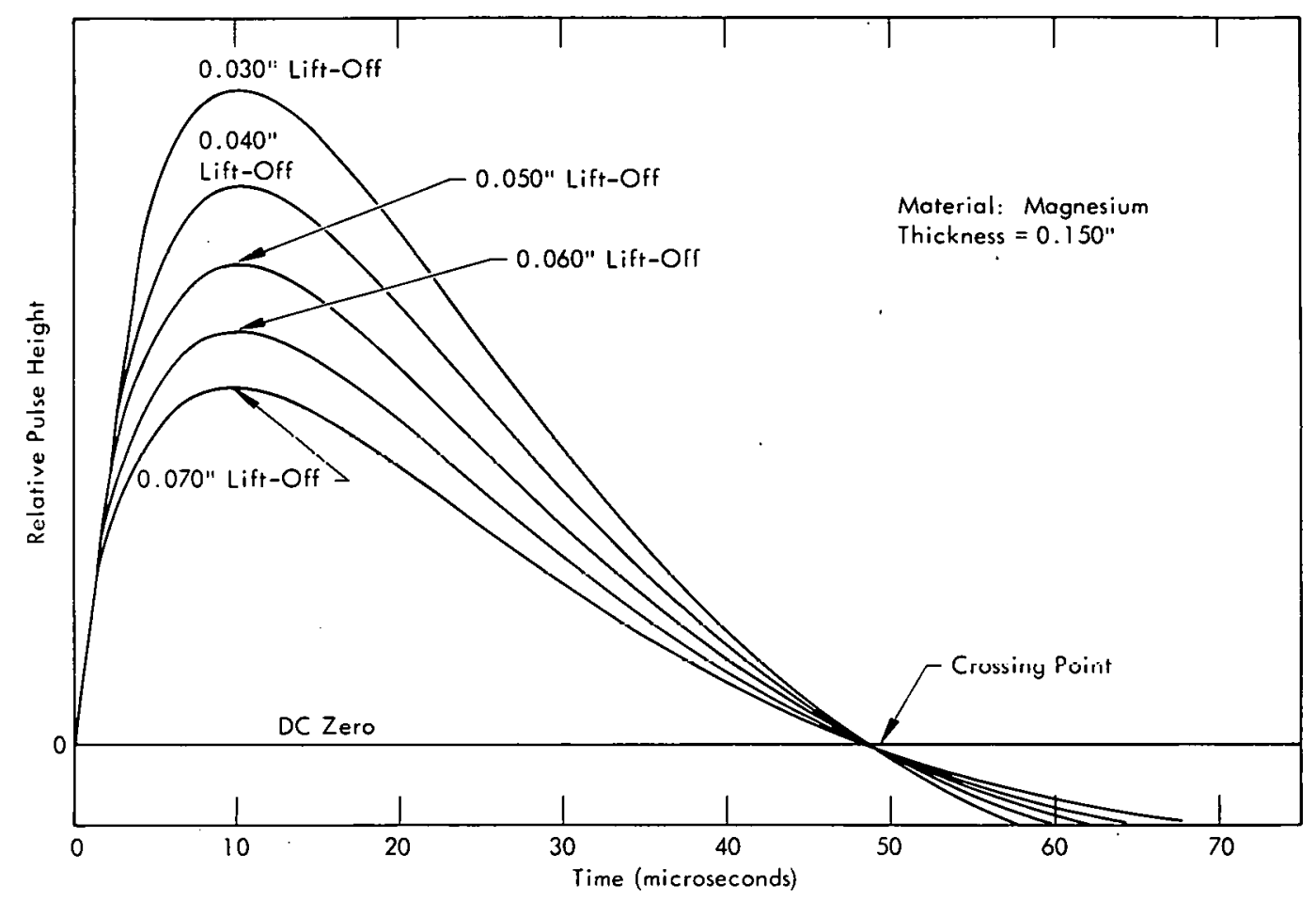

Figure 10. EXPERIMENTAL VOLTAGE PULSE FOR VARIOUS PROBE-TO-METAL SPACINGS.

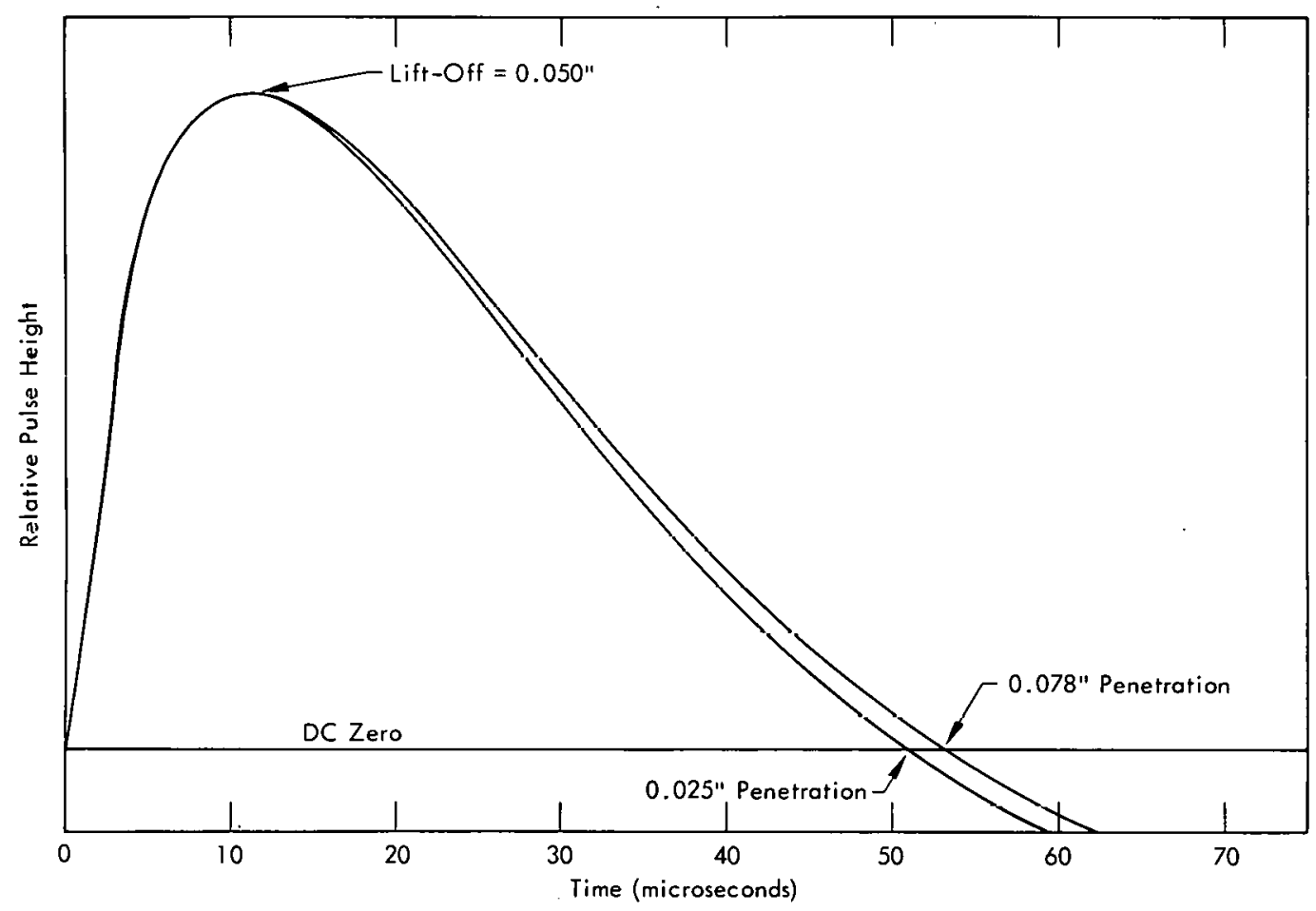

Figure 11. EXPERIMENTAL VOLTAGE PULSE FOR ALUMINUM ELECTRON-BEAM WELDS. 


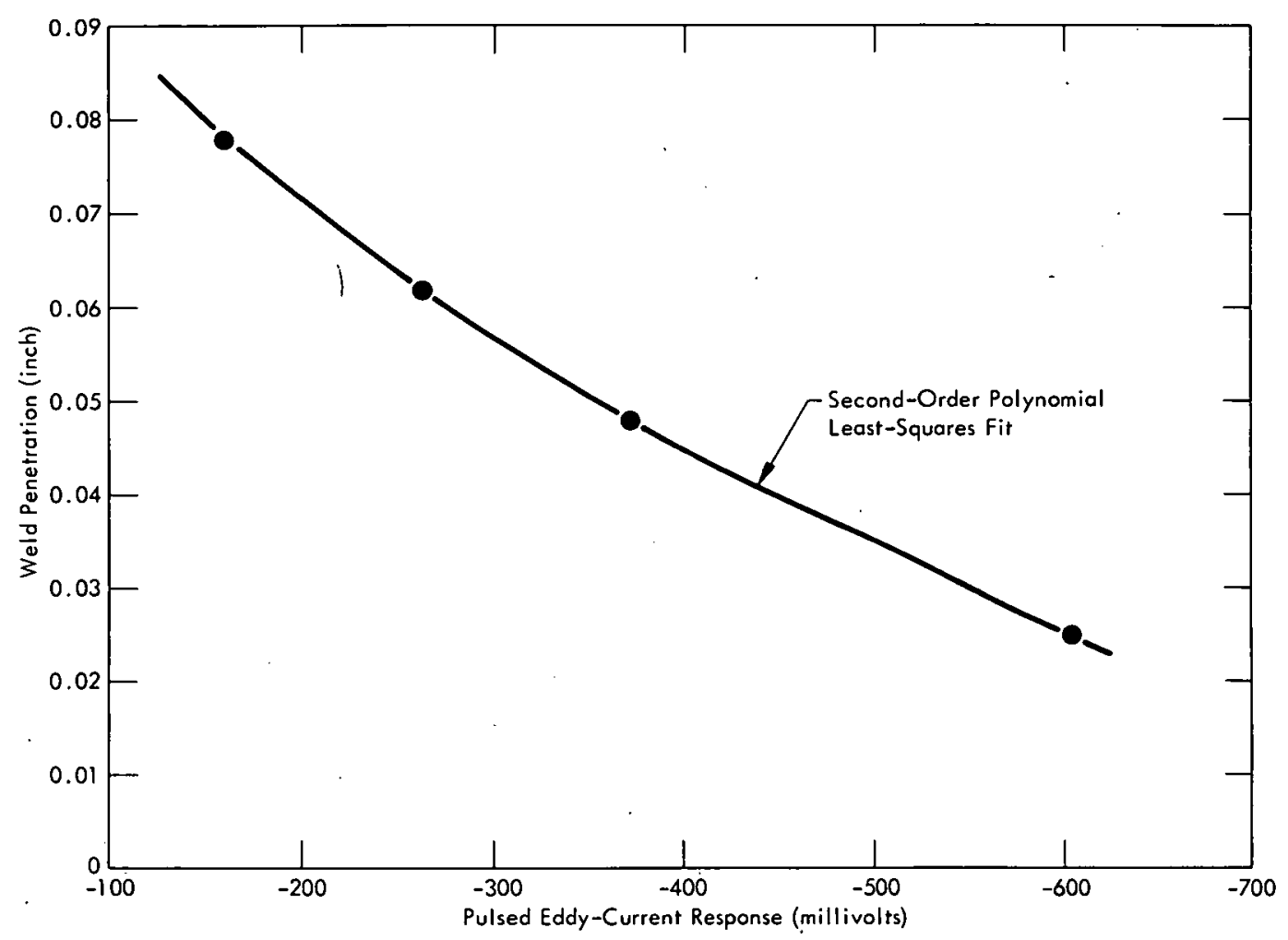

Figure 12. PULSED EDDY-CURRENT RESPONSE FROM ALUMINUM WELD STANDARDS.

peak value of the pulse and keeping it constant. This sampling technique can also be applied to detect small changes in thickness or conductivity of a material.

To demonstrate the continuous scanning capability of the pulsed system where probeto-metal spacing is controlled by sampling the peak value of the pulse and using this sampled signal to drive the liftoff compensating servo-system described above, a strip-chart recording of the response from the electron-beam weld in a 4-inchdiameter aluminum cylinder is illustrated in Figure 13. With the cylinder rotating at a constant speed, the servo-system was able to maintain a constant liftoff of $0.060 \pm 0.0005$ inch even though the part runout was greater than 0.2 inch. The top trace shows the sampled signal at the cross-over point of the pulse when the probe was positioned over the base metal (off the weld) for three revolutions of the cylinder. The bottom trace indicates the relative decrease in the signal level of the weld response at corresponding positions along the circumference of the weld as the penetration depth varies from 0.075 to 0.015 inch. Figure 14 shows the experimental setup used to determine the penetration of the electron-beam weld in this 4-inchdiameter aluminum cylinder.

The sensitivity of the pulsed system to small defects such as voids and porosity in welds was investigated by drilling flat-bottomed holes to a depth of 0.075 inch into the underside of a 0.150 -inch-thick aluminum plate. The diameter of these flat-bottomed holes ranged from 0.020 to $0.090 \mathrm{inch}$, and the response from these 


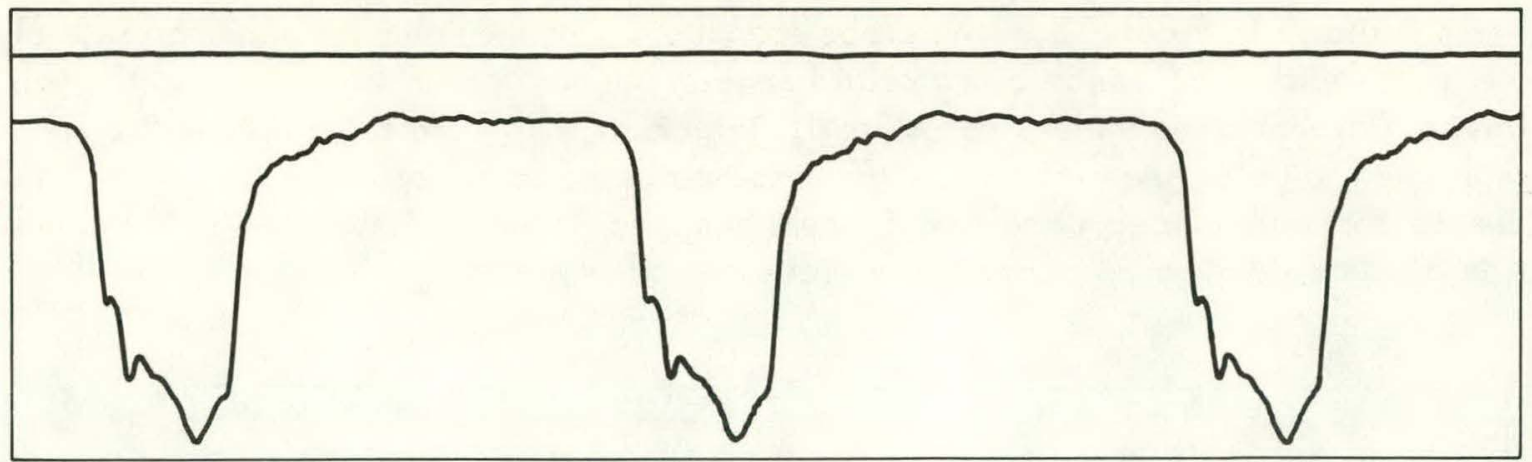

Figure 13. PULSED EDDY-CURRENT RESPONSE FROM AN ELECTRON-BEAM WELD IN A FOUR-INCH-DIAMETER ALUMINUM CYLINDER.

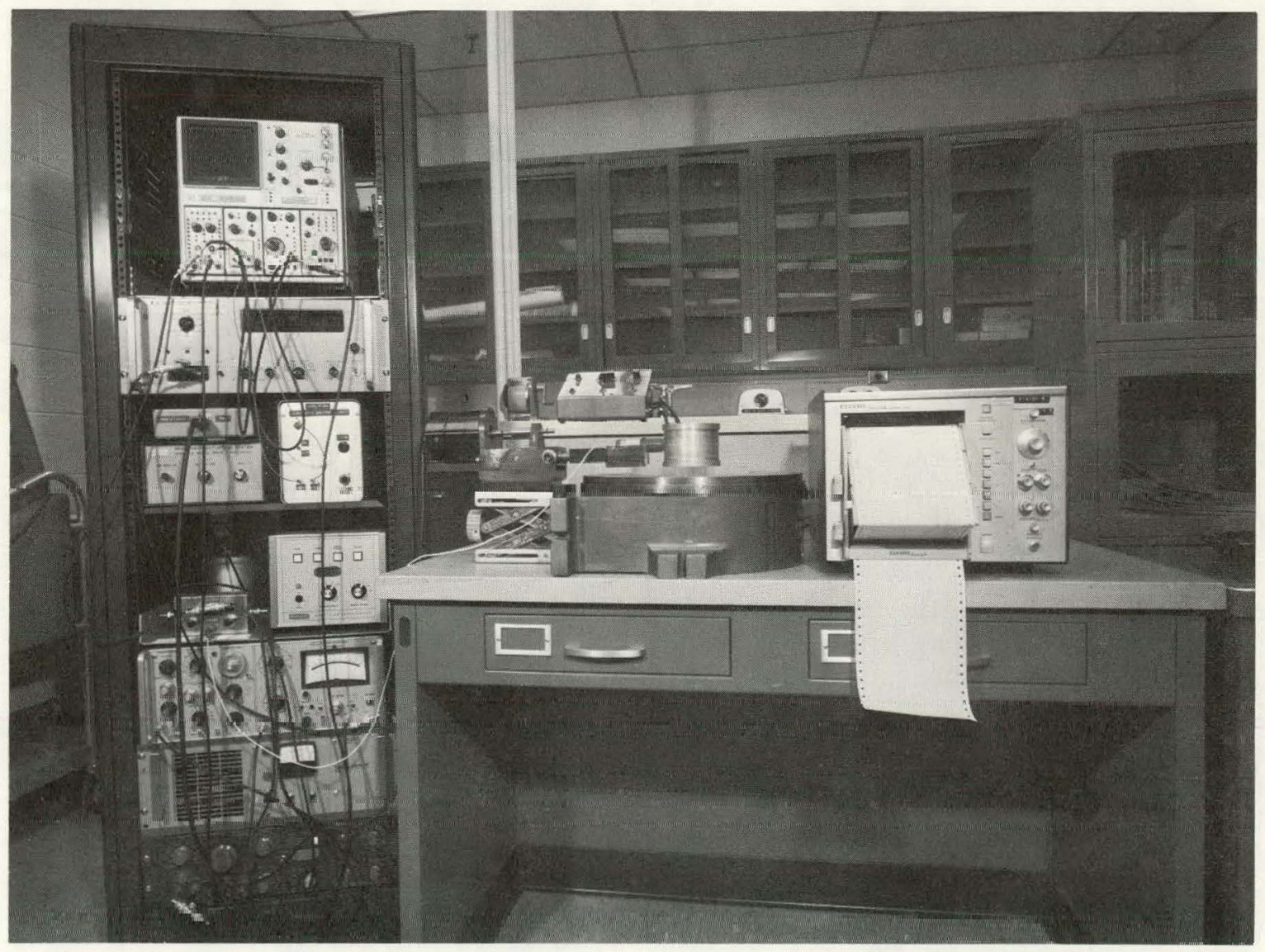

140964

Figure 14. EXPERIMENTAL SETUP FOR TESTING THE PENETRATION OF THE ELECTRON-BEAM WELD IN A FOURINCH-DIAMETER ALIIMINIUM CYIINDER. 
holes is shown in Figure 15 as the probe scanned across the holes from the top side of the plate with 0.075 inch of material between the surface of the probe and the top of the flat-bottomed holes. For a small defect, with an effective diameter of less than the radius of the probe coil, the response has a form similar to the letter "W" due to the unbalanced condition first in one side of the coil and then in the other side as the defect passes beneath the probe.
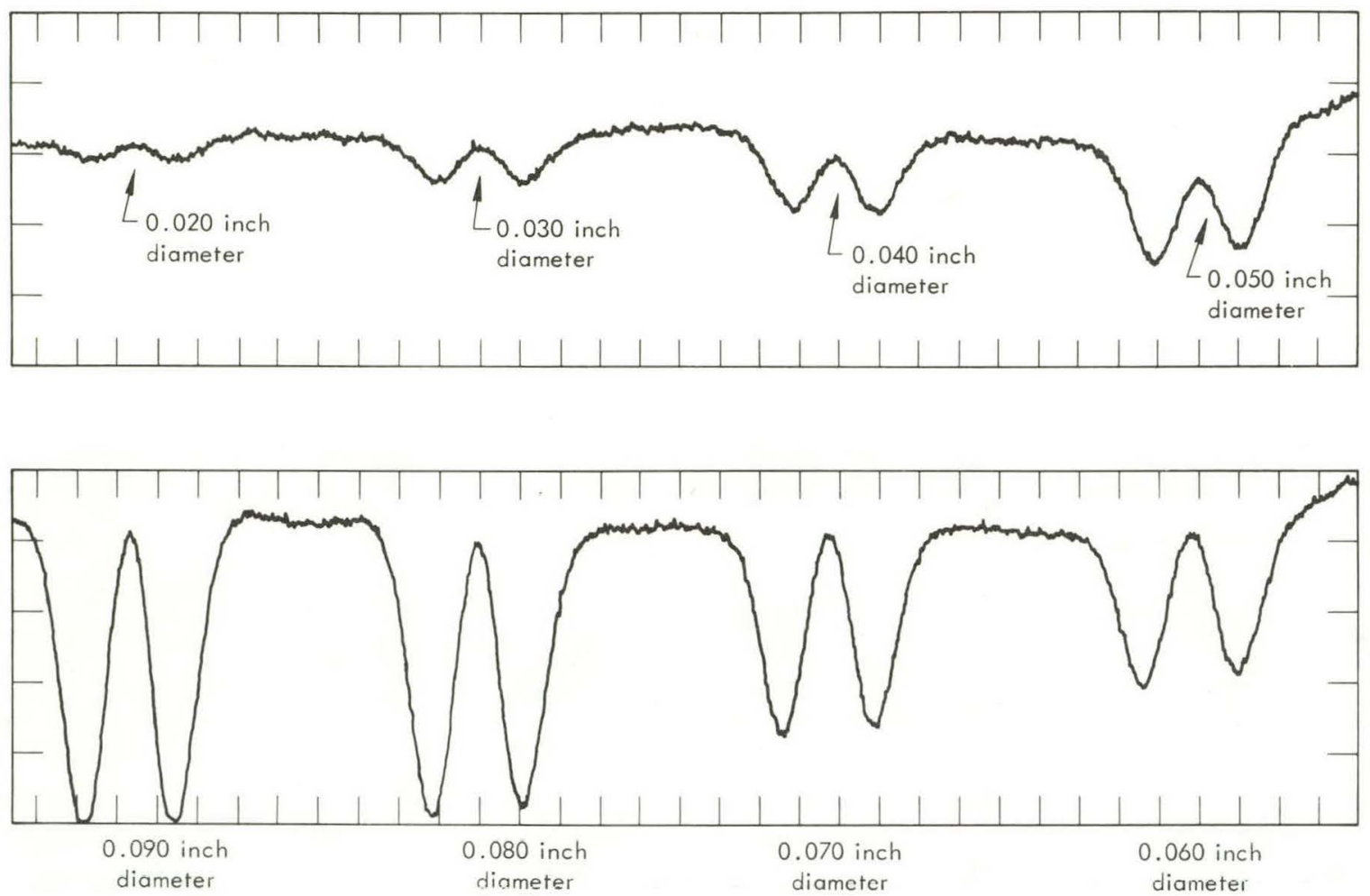

Figure 15. PULSED RESPONSE FROM SCANNING ACROSS FLAT-BOTTOMED HOLES IN AN ALUMINUM PLATE.

As a means of evaluating artificial standards for testing weld penetration, five elox slot standards were fabricated to simulate welds having partial penetrations ranging from 0.018 to $0.030 \mathrm{inch}$. The widths of these elox slots vary from 0.0028 to 0.0051 inch. The ability of the pulsed system to detect small changes (less than 0.002 inch) in elox slot depth is shown in Figure 16. A linear least-squares fit to the five data points results in a standard error of 0.0008 inch.

\section{DISCUSSION}

Although the pulsed eddy-current system appears to be more complicated due to its large number of component parts as compared to most sinusoidal systems, actually the pulsed system is quite easy to set up and use because most of the initial adjustments can be made visually with the aid of the oscilloscope trace. The stability and 


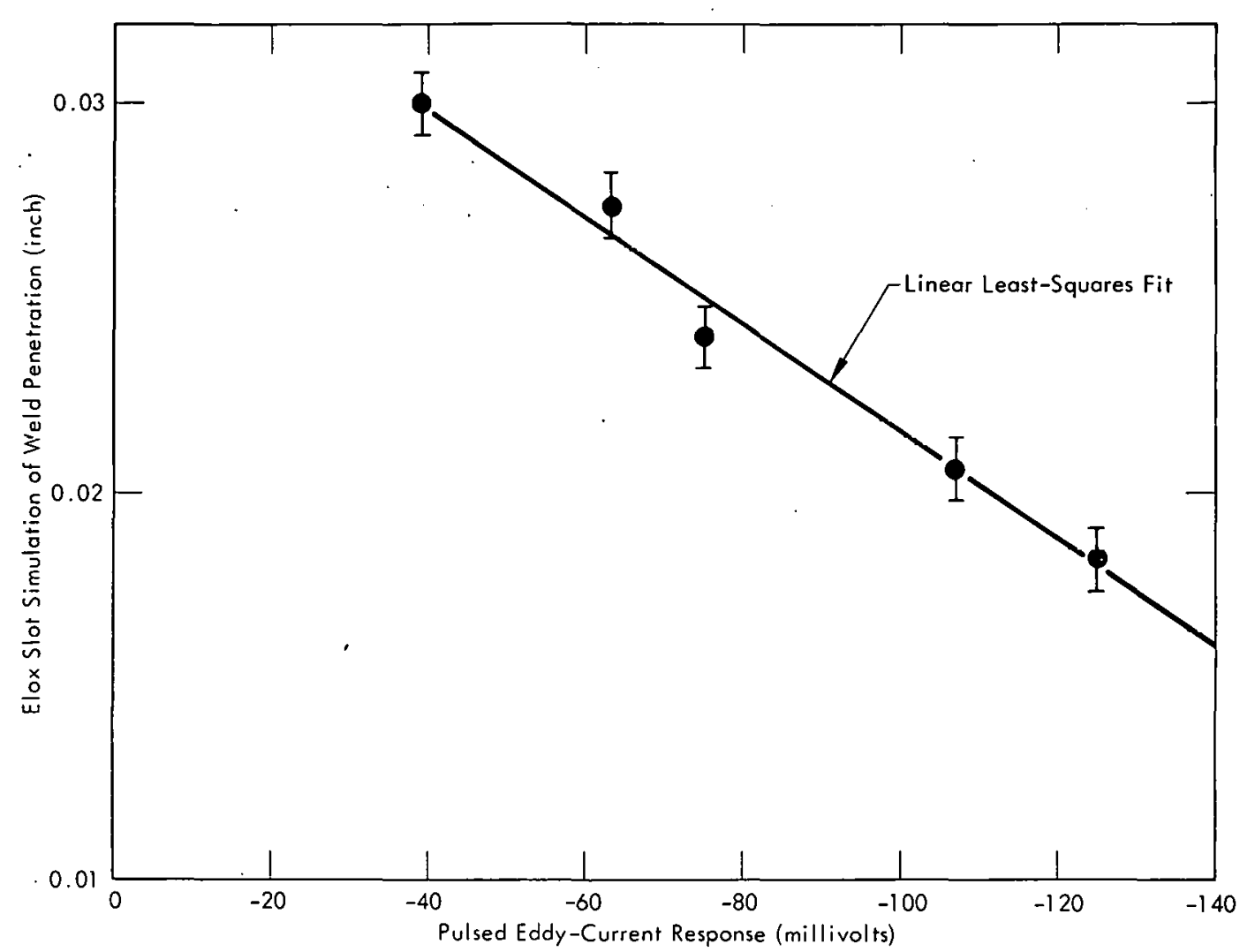

Figure 16. PULSED RESPONSE FROM ELOX SLOTS.

reproducibility of the pulsed system are exceptionally good; and, because of its continuous scanning capability, data taking time is reduced to a minimum. Since the input-pulse power can be varied continuously, the depth-of-field sensitivity is easily controlled. However, the singular important feature of the pulsed system is that more information is contained in a single signal. For example, the front end of the pulse contains the higher frequency components of the pulse-frequency spectrum and is more sensitive to liftoff and surface conditions; whereas, the tail of the pulse, which contains the lower frequency components, is sensitive to defects and variations in thickness and conductivity. The sampling techniques being applied at the present can be extended to the storing of the entire pulse and comparing this stored pulse with calibrated "standard" pulses, which have been stored previously, to extract as much of this information as possible. 


\section{REFERENCES}

(1) Waidelich, D. L., et al; A Pulsed Eddy-Current Technique for Measuring Clad Thickness, ANL-5614; Argonne National Laboratory, Lemont, Illinois; July 1958. 


\section{BIBLIOGRAPHY}

(1) Chan, Shu-Bing; The Penetration, of Pulsed Electromagnetic Fields into a Conductor; University Microfilms, Inc, Ann Arbor, Michigan (1971).

(2) Stratton, J. A.; Electromagnetic Theory; McGraw-Hill Book Company, New York, New York (1941).

(3) Erdelyi, Arthur, et al; Tables of Integral Transforms, Volume I; McGraw-Hill Book Company, New York, New York (1954). 